\title{
A Multifrequency Method for Determining Cochlear Efferent Activity
}

\author{
Anne E. Luebke, ${ }^{1,2}$ Paul K. Foster, ${ }^{1}$ And Barden B. Stagner ${ }^{1}$ \\ ${ }^{1}$ Department of Otolaryngology, University of Miami School of Medicine, Miami, FL 33101, USA \\ ${ }^{2}$ Neuroscience Program, University of Miami School of Medicine, Miami, FL 33101, USA
}

Received: 15 November 2000; Accepted: 10 May 2001; Online publication: 31 August 2001

\begin{abstract}
A test based on measures of distortion-product otoacoustic emissions (DPOAEs) was developed in lightly anesthetized guinea pigs and alert rabbits to assess the effective activation or functional "strength" of the cochlear efferent system. The multifrequency method described here used the DP-gram frequency function to evaluate the fast component of the olivocochlear adaptive effect on DPOAE levels over a 2-octave frequency range. An estimate of any concurrent muscle activation was also determined over the identical frequency range by monitoring the levels of the eliciting $f_{1}$ primary tone throughout its duration. The acoustic reflex, as measured by this $f_{1}$ level constancy test, did not appear to contribute to the average efferent strength of sedated guinea pigs, but the acoustic reflex did contribute to the average "efferent" strength of awake rabbits. Hence, the average efferent effect in alert rabbits is contaminated by the acoustic reflex, which confounds its interpretation.

Keywords: cochlea, distortion-product otoacoustic emissions, fast adaptation, acoustic reflex, olivocochlear efferent system, guinea pigs, rabbits
\end{abstract}

\section{INTRODUCTION}

A detailed knowledge of the functional aspects of the olivocochlear efferent system has been difficult to

Correspondence to: Anne E. Luebke, Ph.D. • University of Miami Ear Institute (M805) - Miami, FL 33101-6960. Telephone: (305) 2434641; fax: (305) 243-5552; email: aluebke@chroma.med.miami.edu obtain, particularly under noninvasive conditions. However, during the last decade, Collet and colleagues (1990) developed a contralateral acoustic stimulation method in humans that enabled investigators to examine, noninvasively, efferent-induced effects on transient-evoked otoacoustic emissions measured from the ipsilateral test ear. These same investigators (CheryCroze et al. 1993; Moulin et al. 1993) later reported that identical contralateral acoustic stimulation in humans caused a similar small reduction of a $\mathrm{dB}$ or so in the levels of distortion-product otoacoustic emissions (DPOAEs). At about the same time, other investigators showed that a similar contralateral acoustic stimulation produced a comparable reduction in the ipsilaterally monitored DPOAEs of the anesthetized guinea pig (Puel and Rebillard 1990). Presumably, such contralateral acoustic stimulation-induced effects on the ipsilateral test ear are mediated primarily by contralaterally responsive olivocochlear projections (the uncrossed efferent system) that are estimated to comprise approximately one-third of the efferent innervation of a given cochlea (Guinan et al. 1983, 1984).

More recently, Liberman et al. (1996) developed a DPOAE-based test in anesthetized cats that assessed the activation of the contralateral, as well as the ipsilateral, olivocochlear projections on what they called the fast-adaptive cochlear response. Specifically, at a single $f_{2}$ of $2 \mathrm{kHz}$, they reported that as a result of an acoustically induced activation of the olivocochlear pathway with long-duration primary tones in the ipsilateral test ear, DPOAEs were maximally reduced at about 0.5-1 $\mathrm{s}$ after the onset of the primaries. Moreover, this adaptive decrease in DPOAEs was greater in response to binaural than to monaural presentations of the primary tones. To ensure that the fast-adaptive response 
they observed was related to olivocochlear efferent system activity, Liberman and colleagues (1996) also showed that this fast-adaptive DPOAE effect was eliminated when the entire efferent nerve-fiber supply to the test ear was severed.

The present study used DPOAEs to examine the influences of the olivocochlear system on outer hair cell responses in two other laboratory species, the sedated guinea pig and alert rabbit. The guinea pigs were lightly sedated as reliable DPOAEs could not be obtained from alert animals. Rabbits can be tested awake with reproducible DPOAEs. The procedure developed by Liberman et al. (1996) was modified to more completely evaluate olivocochlear efferentmediated effects by extending the primary-tone frequencies over a 2 octave test range. In addition to developing this test in the form of an average efferent index, an attempt was made to evaluate the potential contribution of the acoustic reflex to the acoustically induced reductions in DPOAE level, which could be confounding if the long-lasting primary tones also activated the middle ear or other muscles. The major finding was that the acoustic reflex did not contribute to the changes in DPOAE level, and, hence, in the sedated guinea pig, the changes can be assumed to be purely efferent effects. However, this was not the case for the alert rabbit. In that case, the $f_{1}$-constancy test did detect the presence of muscle activity that contributed to the changes in DPOAE level, and hence contributed to the average "efferent" measure in the rabbit. In addition, these average "efferent" measures were highly variable across subjects, i.e., between individual guinea pigs and rabbits, but were more similar within subjects, i.e., between the ears of the same animal, whether sedated or awake.

\section{METHODS}

All procedures performed on both guinea pigs and rabbits were approved by the University of Miami's Institutional Animal Care and Use Committee.

\section{Animal subjects}

Pigmented guinea pigs (strain 2/Ncr, $n=8,300-375$ $\mathrm{g}$, Charles River, MA) and New Zealand pigmented rabbits $(n=7,2-3 \mathrm{~kg}$, obtained commercially from a local supplier) were used as experimental subjects. All animals initially underwent aseptic surgery under general anesthesia (guinea pigs: $40 \mathrm{mg} / \mathrm{kg}$ ketamine $\mathrm{HCl}, 3 \mathrm{mg} / \mathrm{kg}$ xylazine; rabbits: $40 \mathrm{mg} / \mathrm{kg}$ ketamine $\mathrm{HCl}, 10 \mathrm{mg} / \mathrm{kg}$ xylazine) to fix a permanent brace, consisting of an inverted stainless-steel screw headpost, to the dorsal surface of the skull. The headpost supported the animal's head and provided the stability necessary for DPOAE measurements. It also permitted accurate replication of head position upon repeated measurements on different test days. Animals were allowed to recover from the headpost surgery for 3 weeks before the efferent experiments were initiated. During the efferent testing sessions, guinea pigs were lightly sedated with $40 \mathrm{mg} / \mathrm{kg}$ ketamine $\mathrm{HCl}$, whereas rabbits were tested while awake but constrained by a standard rabbit restrainer.

\section{DPOAE measurements}

DPOAEs at $2 f_{1}-f_{2}$ were recorded using an emissions measurement system consisting of commercially available components that included $f_{1}$ and $f_{2}$ earspeakers (Etymotic Research ER-2, Elkgrove Village, IL) and an acoustic probe/microphone assembly (Etymotic Research ER-10B ${ }^{+}$for guinea pigs; ER-10A for rabbits). Stimulus generation and response acquisition were computer-controlled through an on-board digital signal processor operated by customized software as described in detail by Martin et al. (1998). In combination, this system was capable of obtaining DPOAEs in the form of routine DP-grams (i.e., DPOAE levels as a function of frequency) between geometric mean (GM) frequencies of about 1.5 and $16 \mathrm{kHz}$ (i.e., $f_{2}=\sim 1.6-18$ $\mathrm{kHz}$, depending on the $f_{2} / f_{1}$ ratio used), at equivalent 0.1 - or 0.2-octave steps for equilevel primaries ranging from 45 to $75 \mathrm{~dB}$ SPL. These data were used to confirm that the experimental subjects exhibited DPOAEs that were within the normal range based upon the laboratory's database for rabbits and guinea pigs.

\section{Single-frequency fast-adaptive measure}

To initially test for the presence of a single-frequency fast-adaptive response in sedated guinea pigs and alert rabbits, a method was used similar to that developed for anesthetized cats and mice (Liberman et al. 1996; Sun and Kim 1999). That is, monaural or binaural equilevel primary tones of various durations were repeatedly presented to the test ear and averaged two times, as schematized in Figure 1A. DPOAEs were initially sampled for 46-ms-duration primaries. Next, primary tones on times were successively increased prior to the 46-ms sample period. The majority of timepoints were tested with primary tones on times less than $1 \mathrm{~s}$, with 1-s primary-tone durations taken as the point where efferent influences were typically maximal. Because monaural stimulation primarily activates the crossed efferent system, whereas binaural stimulation activates both the ipsilateral and crossed efferent systems (Liberman et al. 1996), there is a greater reduction in DPOAE level observed for the binaural condition. 

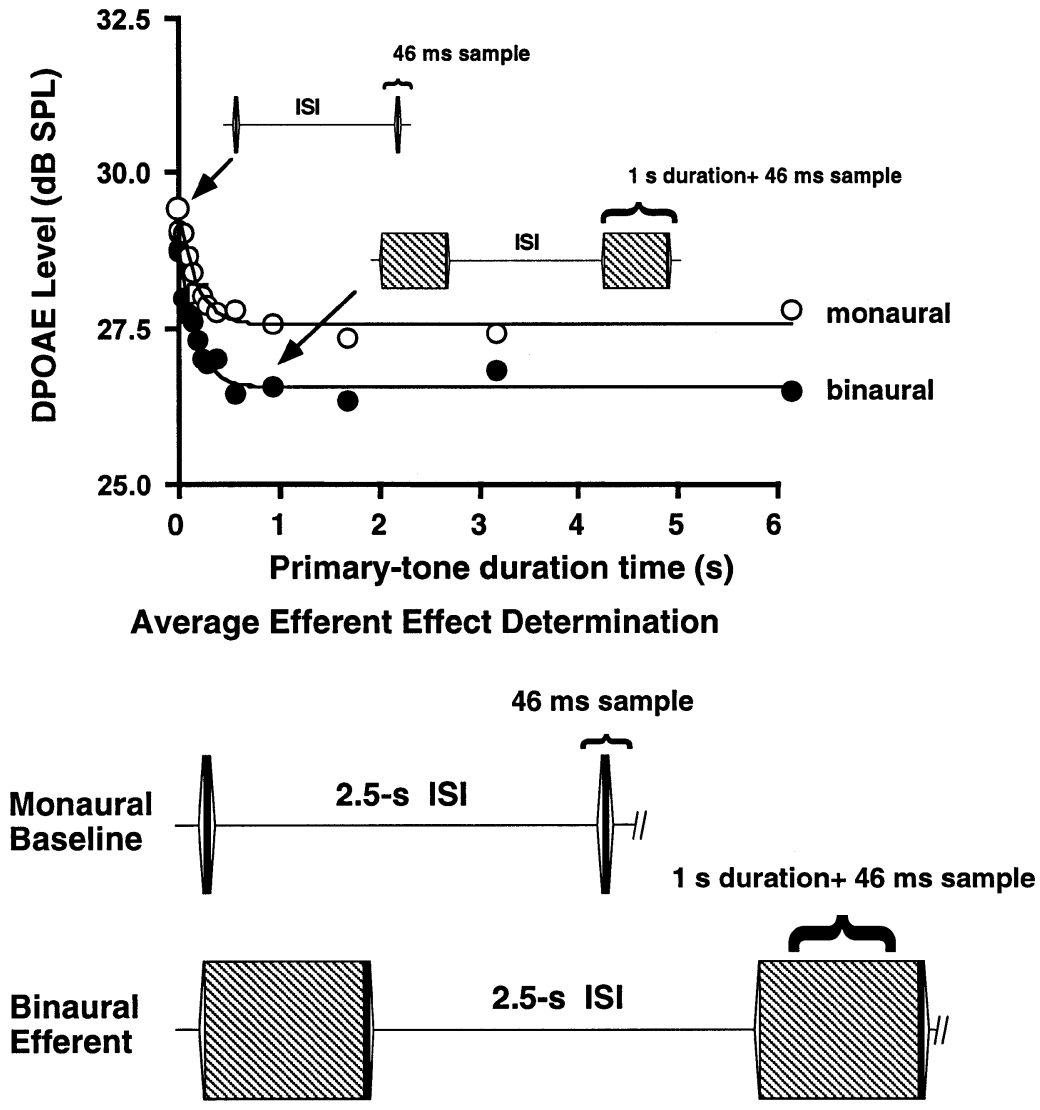

Average Efferent $=\sum_{f_{i}}^{f_{n}} \mid \frac{(\text { Binaural Efferent DPOAE })-(\text { Monaural Baseline DPOAE })_{f_{i}} \mid}{\text { number frequencies tested }}$
FIG. 1. A A schematic showing the single-frequency fast-adaptive measure paradigm. Equilevel primary tones of different durations $(x$ axis) were presented to the test ear. These tones were presented either monaurally $(\bigcirc)$ or binaurally $(\mathbf{)}$. The DPOAEs ( $y$ axis) were measured during the last $46 \mathrm{~ms}$ of the stimulus. Note that 1 -s primary tone durations are the point where efferent influences were typically maximal. B Schematic representation showing the average efferent measurement paradigm. In the monaural baseline condition, DPOAEs were measured over a 46-ms period. In the binaural efferent condition, DPOAE levels were determined during a 46-ms period beginning after a 1-s binaural presentation of $f_{1}$ and $f_{2}$ tones. All tones were presented with a 2.5-s interstimulus interval (ISI). The average efferent measure is the absolute value of the scalar difference between the binaural efferent DPOAE level and the monaural baseline DPOAE level for each frequency $\left(f_{1}\right)$ tested. This difference was then divided by the number of frequencies tested $(n=$ 21 rabbit and $n=11$ guinea pig) to yield the average efferent effect.

\section{Average efferent determination}

The single-frequency fast-adaptive measure was then modified to include only the monaural 46-ms or binaural $1 \mathrm{~s}+46$-ms primary-tone presentations. As schematized in Figure 1B, a baseline paradigm that minimized efferent influences was first performed by measuring emissions monaurally as a DP-gram with 46-ms tone presentations over the 2-octave test range (i.e., at GM frequencies from 2.8 to $11.3 \mathrm{kHz}$ and $f_{2}$ frequencies from $\sim 3$ to $13 \mathrm{kHz}$ ). DPOAE levels were measured at a timepoint that was $5.7 \mathrm{~ms}$ after the ramped onset of the 46-ms primary tones. For guinea pigs, DPOAEs were elicited in 0.2-octave steps ( $n=11$ frequencies) for equilevel primaries at $L_{1}=L_{2}=70 \mathrm{~dB} \operatorname{SPL}\left(f_{2} /\right.$ $\left.f_{1}=1.2\right)$, while for rabbits, a corresponding DP-gram was determined in 0.1-octave intervals $(n=21$ frequencies) for $L_{1}=L_{2}=55$ - $\mathrm{dB}$ SPL primary tones $\left(f_{2} / f_{1}=1.25\right)$, which were previously shown in rabbits to be slightly below the acoustic reflex threshold (Whitehead et al. 1991). Equilevel primary tones were used to ensure that the DPOAE measures would be robust yet not overly sensitive to small fluctuations in DPOAE levels. The $f_{2} / f_{1}$ ratio used in guinea pigs was previously shown to be optimal for DPOAEs obtained in this species (Brown 1987). The guinea pig data were obtained in 0.2- rather than 0.1-octave steps to ensure that the testing could be accomplished within a single 40-minute sedation period.

Following collection of the monaural data, binaural stimulation with primary tones lasting $1 \mathrm{~s}+46 \mathrm{~ms}$ (Fig. 1B) was applied to maximize the efferent-induced adaptive effect. For both the monaural baseline and the binaural efferent tests, each DPOAE measurement was separated by a 2.5 -s interstimulus interval to allow 
sufficient time for any fast-adaptive efferent effects to reset. DPOAE measures were sampled during the last $46 \mathrm{~ms}$ of the $1 \mathrm{~s}+46$-ms stimuli for test frequencies over the same 2-octave extent. The absolute difference scalar values between the DPOAE levels obtained during the baseline monaural and the subsequent binaural stimulation intervals were then computed, summed across the tested frequency range, and divided by the number of frequency intervals in the DP-gram (i.e., 11 for guinea pig and 21 for rabbit) to produce a measure of the average efferent effect, as shown by the equation in Figure 1B. The average efferent metric thus reflected the average amount of activation of the efferent olivocochlear projection to the test ear in terms of its functional effects on DPOAE level, over a 2-octave frequency range.

\section{Average acoustic reflex determination or $f_{1}$-constancy test}

In an attempt to estimate the effect the middle-ear muscles, i.e., the acoustic reflex, had on the efferent response, a measure of the average acoustic reflex was also computed. Muscle activity that could alter middleear impedance could be monitored by changes in the level of the $f_{1}$ primary tone in the outer-ear canal. Similar to the computations performed to establish the average efferent value, the difference between the levels of the $f_{1}$ tones measured during the baseline monaural compared with the subsequent binaural stimulation periods was computed, summed across the identical 2-octave frequency range as the average efferent index, and averaged by dividing by the relevant number of tested frequencies in the DP-gram. The relationship between the average efferent index in the right and left ears was analyzed using commercial software (StatView v. 4.5, Abacus Concepts, Inc., Berkeley, CA) to determine linear regression coefficients and $p$ values.

The average efferent and average acoustic reflex measures were determined in seven guinea pigs under ketamine sedation, with five animals tested bilaterally ( $n=12$ ears). Similarly, efferent and acoustic reflex measures were determined for six alert rabbits, with five animals tested bilaterally (11 ears).

\section{RESULTS}

The fast-adaptive method developed by Liberman et al. (1996) to test olivocochlear function in the anesthetized cat was modified for application to the sedated guinea pig and alert rabbit using both monaural and binaural primary-tone stimulations. However, before presenting the average multifrequency efferent effects, typical examples of the effects of this protocol at single frequencies are shown in Figure 2. The plot

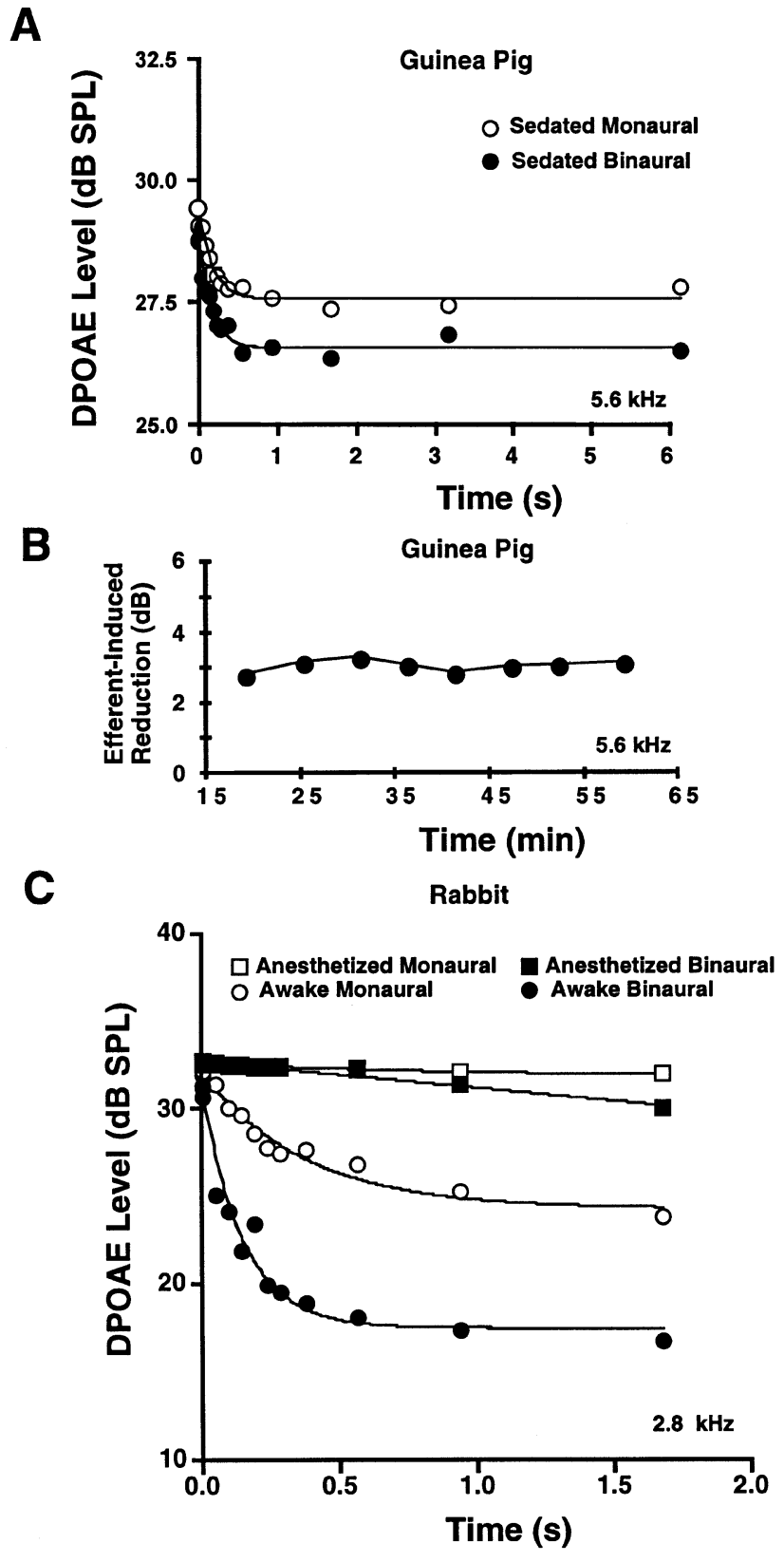

FIG. 2. Efferent-induced fast-adaptive effects on DPOAEs in the guinea pig and the rabbit at single test frequencies. A Binaural auditory stimulation $(-)$ at $\mathrm{GM}=5.6 \mathrm{kHz}$ or $f_{2}=6.2 \mathrm{kHz}\left(L_{1}=L_{2}=65 \mathrm{~dB}\right.$ $\mathrm{SPL})$ produced a greater reduction in DPOAEs $(\sim 3 \mathrm{~dB})$ than did monaural stimulation $(\sim 2 \mathrm{~dB})$. Note efferent reduction in DPOAE levels are maximal after $\sim 1 \mathrm{~s}$ of continuous primary-tone stimulation. B The fast-adaptive binaural response was repeatable around a mean of $3.1 \pm 0.2 \mathrm{~dB}(1 \mathrm{SD})$, as shown here for a series of measures that were repeated at 5 -min intervals over about a 50 -min period at 5.6 $\mathrm{kHz}$ for the same sedated guinea pig shown in A. C Using a GM test frequency of $2.82 \mathrm{kHz}\left(f_{2}=3.16 \mathrm{kHz}\right)$ with $L_{1}=L_{2}=65 \mathrm{~dB} \mathrm{SPL}$, binaural $f_{1}$ and $f_{2}$ stimulation in an alert rabbit $(\mathbf{O})$ produced a greater adaptation $(\sim 12 \mathrm{~dB})$ than a corresponding monaural $(\bigcirc)$ stimulation $(\sim 6 \mathrm{~dB})$. As indicated by the square-symbol functions at the top of this plot, ketamine/xylazine anesthesia greatly reduced the efferentinduced effects in the anesthetized rabbit, whether tested monaurally (open squares) or binaurally (filled squares). 


\section{Guinea Pig}

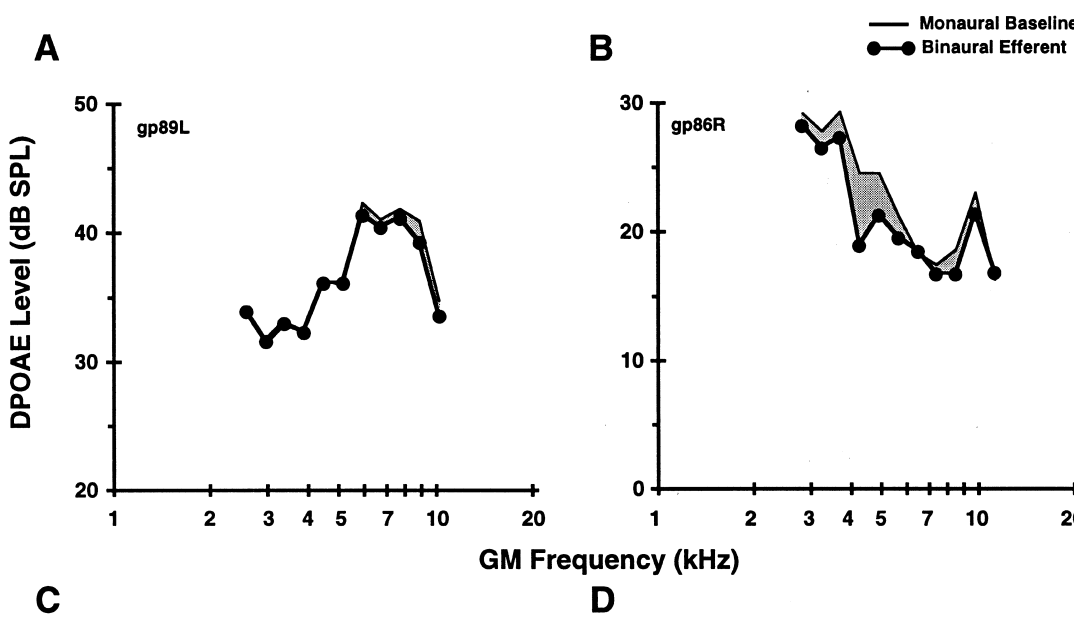

FIG. 3. The average efferent index was highly variable across animals, as shown here for two representative guinea pigs. A, B Shaded regions show, for two sedated guinea pigs $[\mathrm{A}=$ gp89L ( $\mathrm{L}$ $=$ left ear), $\mathrm{B}=\operatorname{gp} 86 \mathrm{R}(R=$ right ear $)]$, the con-
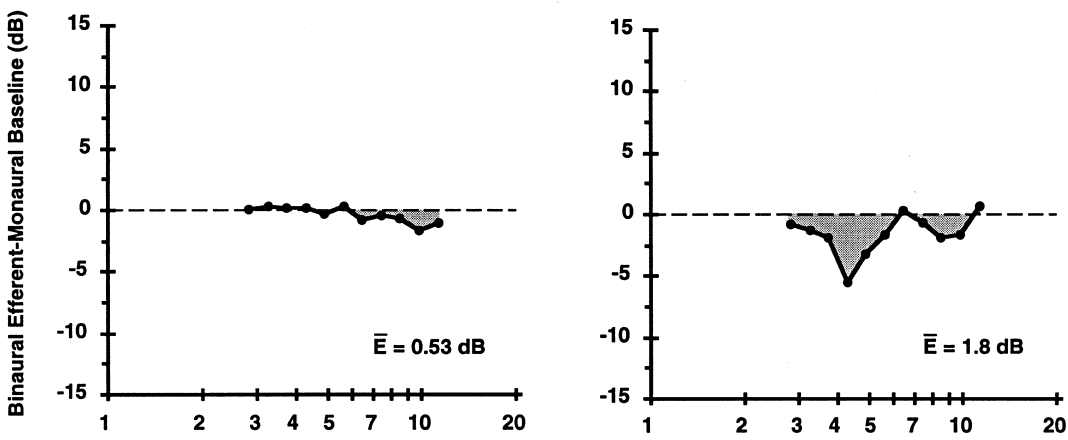

GM Frequency (kHz) trasts in absolute DPOAE levels elicited by baseline monaural (-) vs. binaural efferent stimulation (-) for GM frequencies spanning the same 2-octave frequency interval $\left(L_{1}=L_{2}=\right.$ $70 \mathrm{~dB}$ SPL). For these and all other average efferent tests, noise-floor levels (not shown) were $<-10$ dB SPL. C, D Difference values between binaural efferent and monaural baseline tests (shaded regions in $\mathbf{A}$ and $\mathbf{B}$ ) are shown here for the same representative guinea, pig ears $(C=$ gp89L, $D=$ gp86R). The average of the absolute values of the differences in $\mathrm{dB}$ between the binaural efferent and monaural baseline stimulation, represented by the average efferent $(\bar{E})$ index (see text), is indicated in the lower-right corner of each plot.

in Figure 2A shows that, similar to the anesthetized cat, the olivocochlear-induced reduction in DPOAE levels in the sedated guinea pig was greater for binaural (filled circles) than for monaural (open circles) primary-tone stimulation. That this fast-adaptive effect of olivocochlear activation on DPOAE level was very repeatable within the same test session is shown by the data plotted in Figure 2B, which illustrates, for a sedated guinea pig, test/retests that were performed every $5 \mathrm{~min}$ over an approximately 50-min period. Although not shown here, the primary-tone-induced reductions in DPOAEs also demonstrated test/retest repeatability when the same animal was re-examined 2 days later. In the rabbit, general anesthesia with a mixture of ketamine $\mathrm{HCl}$ and xylazine drastically diminished the olivocochlear-mediated and acoustic reflex-mediated reductions in DPOAEs, as shown by the square-symbol functions in Figure 2C, even though baseline DPOAEs were stable.

The magnitude of the awake rabbit's efferent adaptive response, when compared with the much smaller changes observed in the sedated guinea pig, could potentially reflect the combined influences of the efferent system and the primary-tone-induced acoustic reflex. Because efferent and acoustic reflex responses are difficult to separate in the alert animal, all subsequent tests of efferent function in the awake rabbit, as measured with the average efferent test, used equilevel 55-dB SPL primary tones to reduce the likelihood of eliciting the acoustic reflex (Whitehead et al. 1991).

The plots of Figures 3A and B illustrates the efferent effect on the DP-gram at $L_{1}=L_{2}=70 \mathrm{~dB}$ SPL for the left and right ears of two representative guinea pigs (i.e., gp89L and gp86R, respectively) by displaying both the monaural baseline and binaural efferent DPgrams. The corresponding difference plots (i.e., binaural efferent minus monaural baseline DP-gram levels) for these same guinea pigs are shown in Figure 3C and D, respectively, with the computed average efferent value $(\bar{E})$ indicated at the lower right of each plot.

Similarly, Figure 4A and B display comparable DPgram findings for the right ears of two typical alert rabbit subjects (i.e., r44R and r45R). Similar to the guinea pig plots of Figure 3, the corresponding difference plots for these rabbits are shown in Figure $4 \mathrm{C}$ and $\mathrm{D}$ with the computed average efferent value $(\bar{E})$ indicated at the lower right of each plot. Together, the 
Rabbit

A

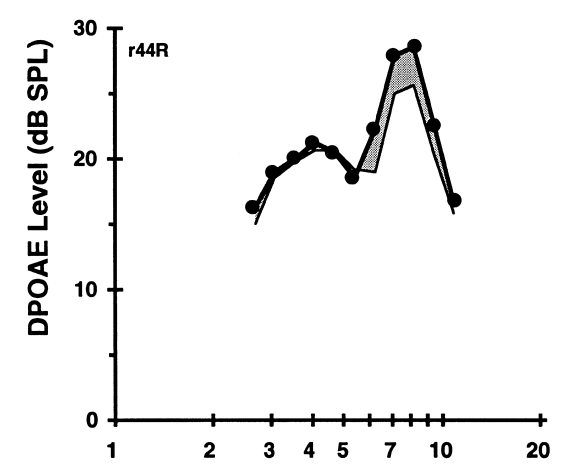

B

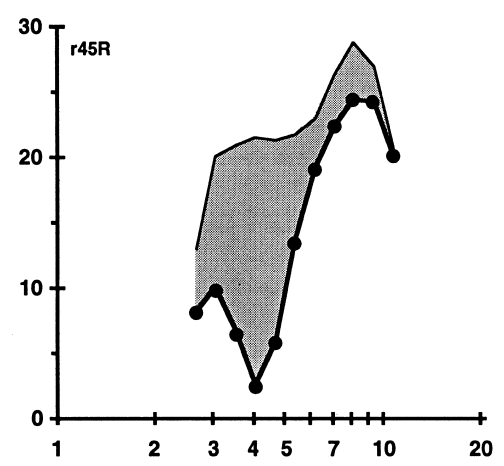

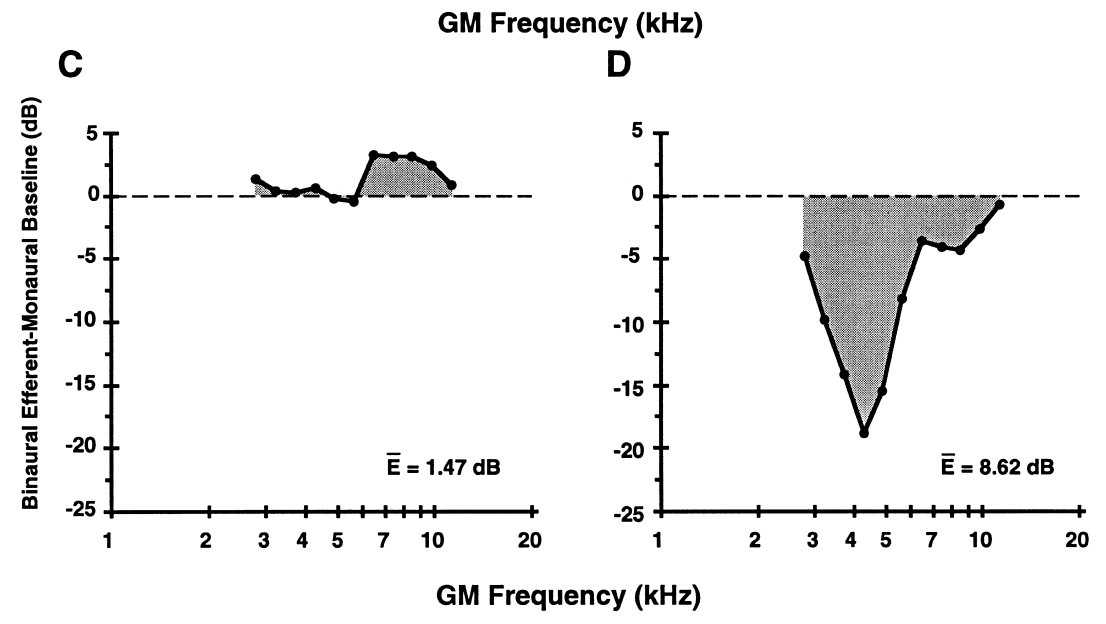

FIG. 4. The average efferent index was highly variable across different rabbits shown here for two representative cases. A, B Shaded regions show, for two ears from different alert rabbits (A $=r 44 R, B=r 45 R$ ), the absolute DPOAE levels elicited by baseline monaural (-) vs. binaural stimulation conditions (-) for GM frequencies tested over a 2-octave interval. C, D The difference values between binaural efferent and monaural baseline tests (shaded regions in $\mathbf{A}$ and B) are shown here for the same rabbit ears ( $C=$ $r 44 R, D=r 45 R)$. The average of the absolute values of the differences in $\mathrm{dB}$ between the binaural efferent and monaural baseline stimulation, represented by the average efferent $(\bar{E})$ index (see text), is indicated in the lower-right corner of each plot. four subjects shown in Figure 3 and 4 demonstrate the typical variability observed between efferent effects as measured by the average efferent method in different animals of the two species. Also, note that awake rabbits (e.g., Fig. 4D), in general, showed larger changes in DPOAE levels than ketamine-sedated guinea pigs.

The plots of Figure 5 show examples of the average acoustic reflex measures for the same animals as in Figures 3 and 4 . The average acoustic reflex is illustrated only as a difference plot between the binaural efferent and monaural baseline stimulation conditions. It is evident from the plots of Figures $5 \mathrm{~A}$ and $\mathrm{B}$ that in ketamine-sedated guinea pigs with 70-dB SPL equilevel primaries, only small changes were detected between the monaural vs. binaural $f_{1}$ levels. Thus, the acoustic reflex likely did not contribute a great deal to the average efferent measure in sedated guinea pigs.

However, the awake rabbits exhibited an acoustic reflex in response to the 55-dB SPL primary-tone stimulation, as indicated by the changes in $f_{1}$ levels shown in Figures 5C and D. Note, however, that the acoustic reflex index is represented by a difference in the levels of the $f_{1}$ primaries, whereas the average efferent effect is given by the difference in DPOAE levels, so that the two sets of values are not directly comparable.

Figures $6 \mathrm{~A}$ and $\mathrm{C}$ compare the magnitude of both the average efferent (shaded bars) and average acoustic reflex (striped bars) measures for all ears tested, with most animals having been tested bilaterally: Two bars are displayed for such subjects, with the left-hand bar representing the left ear and the right-hand bar representing the right ear. In Figure 6A, showing data for sedated guinea pig ears, the acoustic reflex was small, as demonstrated earlier in Figure 5 for example.

Figure 6C plots the efferent and acoustic reflex indexes for all rabbit ears tested. It is clear that, in awake rabbits, an average acoustic reflex (striped bars) varied from $<1$ (e.g., \#65) to over $4 \mathrm{~dB}$ (e.g., \#45). The magnitude of the average acoustic reflex in alert rabbits suggests that the acoustic reflex affected the corresponding average "efferent" measure. Thus, the rabbits with large efferent indexes tended to have the larger acoustic reflex indexes (e.g., r45), and, not surprisingly, the efferent and acoustic reflex values were correlated ( $R=0.824, n=11$ ears, $p<0.002)$, further suggesting that the contribution of the acoustic reflex 


\section{Guinea Pig}

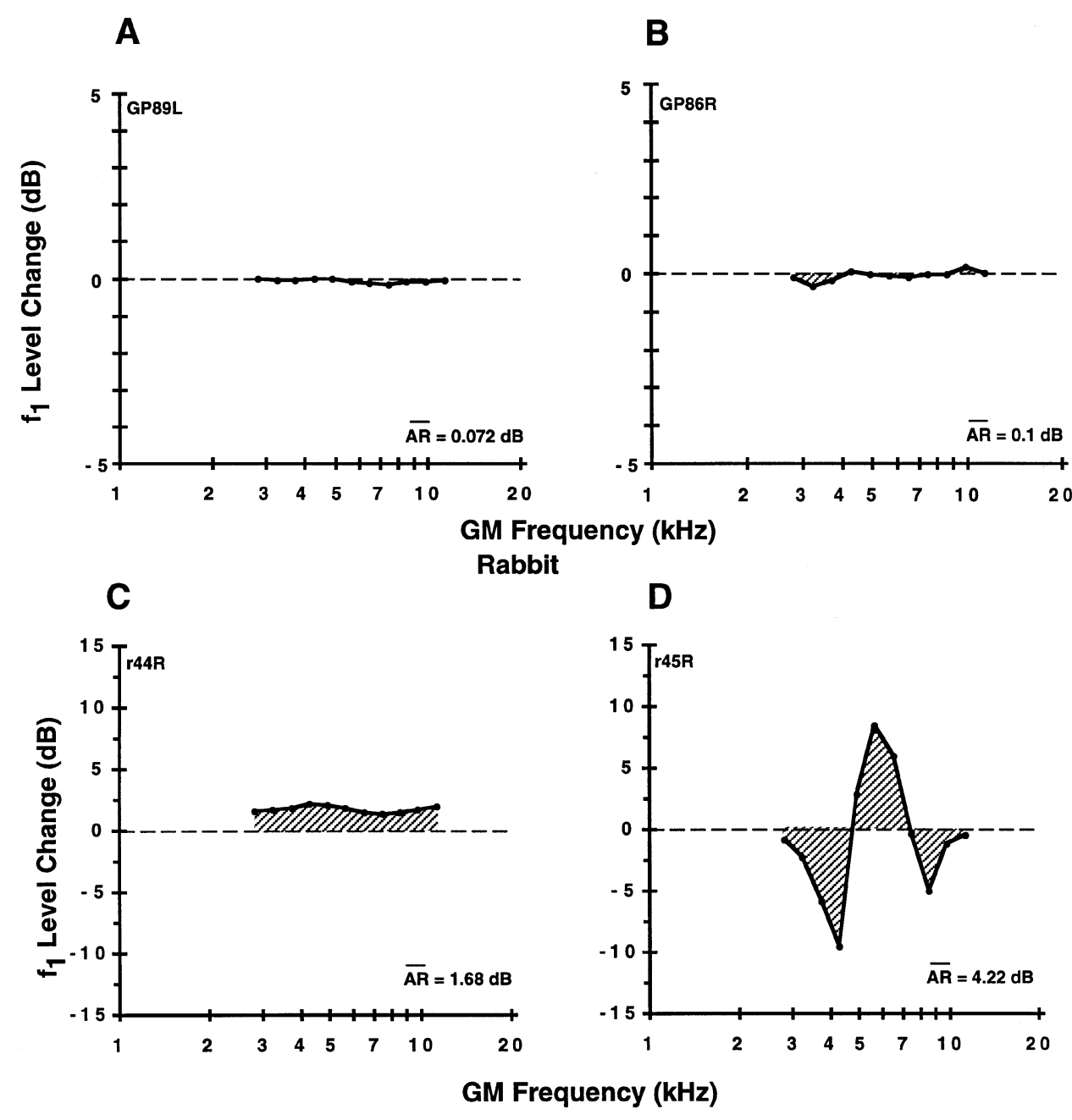

FIG. 5. The $f_{1}$-constancy test or "average acoustic reflex" method that estimated the strength of the acoustic reflex based upon changes in sound pressure in the test ear during the binaural efferent and monaural baseline test conditions. A, B Difference plots for the same guinea pig ears as in Fig. 3 , between the $f_{1}$ tones in the presence of binaural efferent (1.046-s duration) vs. monaural baseline (0.046-s duration) sound stimulation. If the acoustic reflex was activated by the longer $\left(L_{1}=L_{2}=70 \mathrm{~dB} \mathrm{SPL}\right)$ binaural tones, the average acoustic reflex $(\overline{A R})$ magnitude would be significant. C, D In contrast, for alert rabbits, the average acoustic reflexes (AR) for the same ears as shown in Fig. 4 indicated that some acoustic reflex-related activity was evoked in response to the 55-dB SPL primary tones. Note that the $f_{1}$ levelchange scale ( $y$ axis) is tripled for the alert rabbit data. in the rabbit influenced and contaminated the average "efferent" or "feedback" measure.

For animals tested bilaterally, a tendency for the average "efferent" or "feedback" measure from the two ears to be somewhat correlated was observed, especially for rabbits $\left(R^{2}=0.95, n=5\right.$ animals, $\left.p=0.005\right)$ as shown in Figure $6 \mathrm{D}$ and for guinea pigs as shown in Figure 6B.

\section{DISCUSSION}

The present results demonstrate that a reasonable portion of the frequency range of several common laboratory species can be assessed for the capacity of the olivocochlear efferent system to influence cochlear activity as measured by DPOAEs. In addition, the observed findings indicate that the general adaptive efferent effect in sedated guinea pigs and alert rabbits, as measured here at multiple frequencies, was similar to that described earlier (Liberman et al. 1996) for the anesthetized cat at a single frequency. That is, the olivocochlear-induced reduction in DPOAE levels was stable over time and repeatable. Liberman et al. (1996) showed that the adaptive efferent effect on DPOAEs was eliminated when the efferent fibers were severed. While there was no attempt to eliminate the efferent fibers in this study, experiments were performed using xylazine, which affects $\alpha_{2}$ adrenergic receptors present on neurons of the brainstem and results in a decreased efferent and presumably a reduced acoustic reflex for rabbits as shown in Figure 2C (Smith and Mills 1989; Asti et al. 1996; Harel et al. 1997).

More specifically, lightly sedated guinea pigs showed average efferent responses that varied from $<1$ to almost $4 \mathrm{~dB}$, whereas alert rabbits exhibited a variability that ranged from $\sim 1$ to almost $9 \mathrm{~dB}$. This possibly reflects the combined influences of the efferent and the acoustic reflex contributions. While these numbers are not large for the sedated guinea pig, they are averages of the efferent-induced DPOAE level changes per frequency, with the tested frequencies 

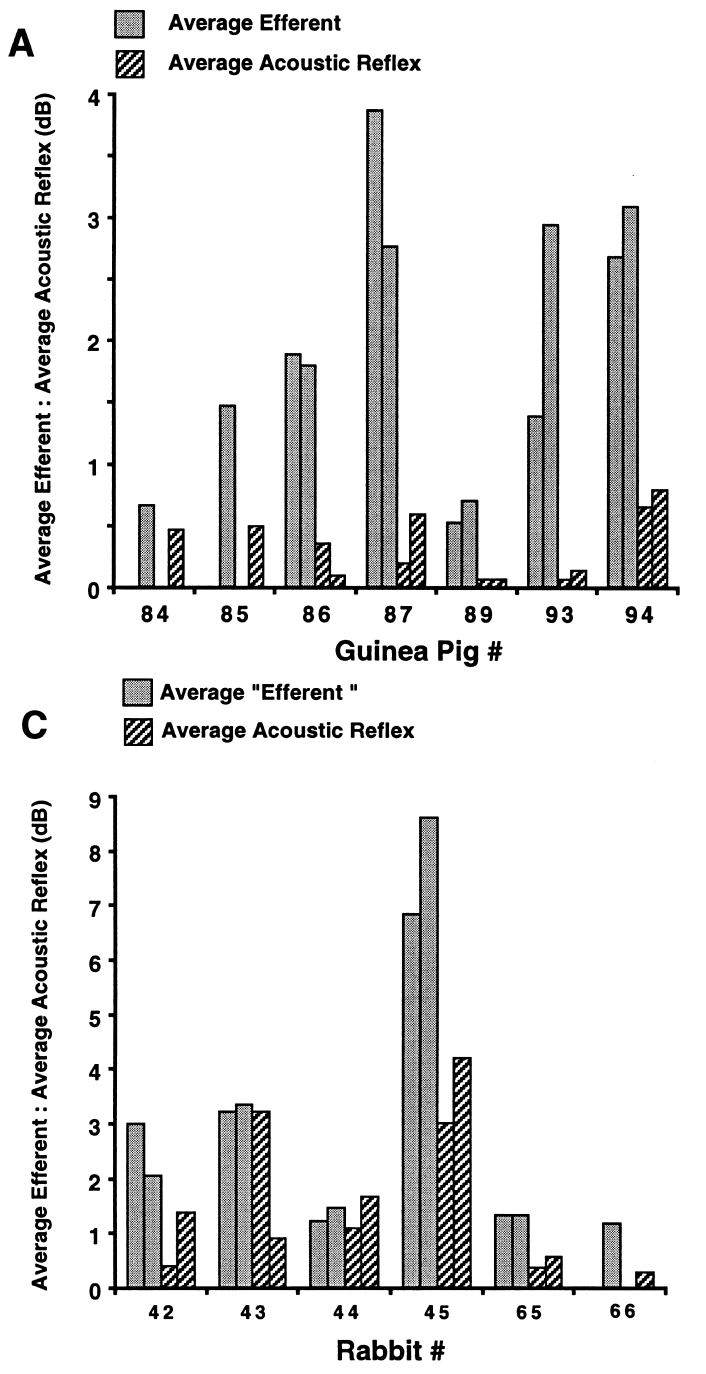

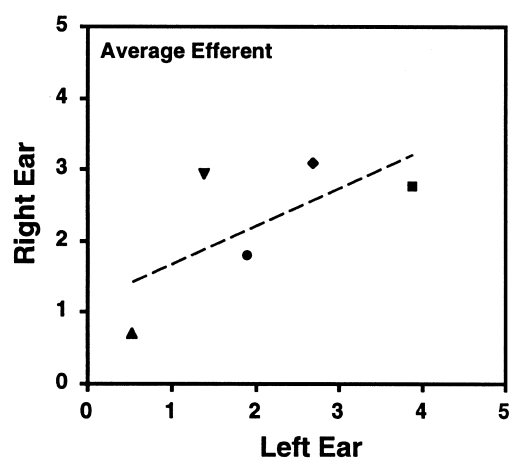

D

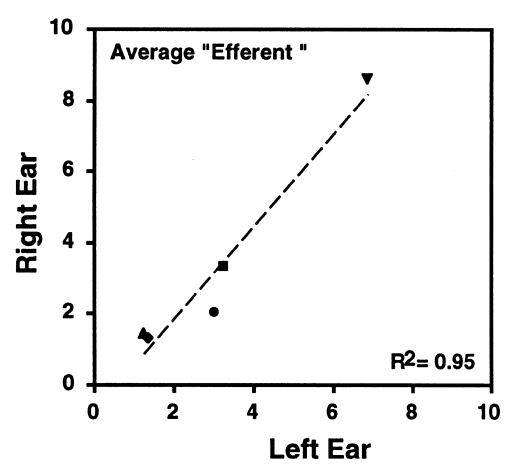

FIG. 6. The average efferent effect (shaded bars) and the average acoustic reflexes (striped bars) are shown for all preparations. Bars on the left represent measures from the left ear, while those on the right indicate values obtained from the right ear. A Note that the guinea pig's average efferent values ranged from $<1$ to $\sim 4 \mathrm{~dB}$. B The magnitude of the efferent response for the right ear of guinea pigs was only generally related to the corresponding left ear value $(n=5)$. C Unlike sedated guinea pigs, alert rabbits had average acoustic reflexes ranging from 1 to about $4 \mathrm{~dB}$, which indicated that acoustic reflex-related activity was present, even at low primarytone levels of $55 \mathrm{~dB} \mathrm{SPL}$, and hence the average efferent effect is influenced by the presence of an acoustic reflex. Note the $y$-axis scale is doubled for the alert rabbit data. D In alert rabbits, there was a strong correlation between the combined "efferent" effects of the right and left ears $\left(R^{2}=0.95, n=5\right.$ ears, $p=0.005)$. distributed over a 2-octave range. Earlier studies found $\sim 6 \mathrm{~dB}$ to be the maximal change in DPOAE levels when direct electrical stimulation of efferent fiber tracts was used to electrically activate the efferent system in both guinea pigs and chinchillas (Mountain et al. 1980; Siegel and Kim 1982). Moreover, our numbers are consistent with earlier studies of the magnitude of efferent-induced effects on evoked otoacoustic emissions in awake humans also measured over a specific multifrequency extent (Berlin et al. 1994).

For sedated guinea pigs, the average acoustic reflex was minimal, probably because of the ketamine sedation. However, for alert rabbits, the average acoustic reflex values that ranged from $<1$ to about $4 \mathrm{~dB}$ suggested that an acoustic reflex was present, even at the low primary-tone levels of $55 \mathrm{~dB}$ SPL, which is below the previously determined 60-dB SPL threshold for the monaural acoustic reflex in alert rabbits (Whitehead et al. 1991).

Maison and Liberman (2000) recently reported on the results of using a monaural single-frequency
DPOAE-based measure of efferent activity in alert guinea pigs, where the unequal primary-tone levels were varied relative to each other, between 60 and 80 dB SPL, at about 5-dB steps (Maison and Liberman 2000). These investigators noted efferent-induced effects ranging from 4 to $14 \mathrm{~dB}$ at $f_{2}=10 \mathrm{kHz}$. However, it is possible that this unequal primary-tone level test detected efferent-induced changes via two-source cancellations or notches in the DPOAE function which would magnify the efferent effect. In alert rabbits, such notches have been shown to be elicited more frequently by nonequal rather than by equilevel primary tones (Whitehead et al. 1992).

In addition, Maison and Liberman (2000), using a monaural single-frequency DPOAE-based measure of efferent strength in alert guinea pigs, found that the variability they observed predicted susceptibility to acoustic overexposure. Thus, awake guinea pigs with larger efferent effects showed less noise-induced threshold shifts than did animals with smaller efferent measures. In their experiments, however, the change 
in ear-canal sound pressure, which likely reflects acoustic reflex-related activity, was not deliberately monitored, so it was not clear what proportion of the protection from noise exposure was simply due to a strong acoustic reflex; however, the threshold for activation of the monaural acoustic reflex is higher than binaural activation. Other studies in which the middle-ear muscles were sectioned suggest that the acoustic reflex was not responsible for these protective effects in the guinea pig. The variability in the average feedback effects observed in the current study for pigmented guinea pigs $(2 / \mathrm{Ncr})$ and rabbits (New Zealand) of the same strain is notable. It is noteworthy though that although guinea pig and rabbit ears showed variability in both the acoustic reflex and the amount of efferent-related activity measures, the magnitude of the average efferent index was correlated between the right and left ears of the same animal, especially for rabbits, as shown in Figure 6D.

Because the average efferent can be influenced by muscle activity, as has been shown in this study, it is important that some index of muscle activity be monitored whenever the cochlear efferent system is tested in alert subjects, whether humans or animals. Another possible influence on DPOAE levels is stimulus-frequency otoacoustic emission (SFOAE) which could also modify the resulting emission. SFOAE has never been detected in the alert rabbit (Martin, personal communication), yet this type of emission is present in the human cochlea and could prove to be another confounding factor in the interpretation of sound-evoked DPOAE level changes.

The relative contributions of the acoustic reflex and efferent effects in the alert rabbit are not yet known. The binaural acoustic reflex threshold, assayed using the $f_{1}$-constancy test, for one lightly sedated rabbit (ketamine $40 \mathrm{mg} / \mathrm{kg}$, acepromazine 1 $\mathrm{mg} / \mathrm{kg}$ ) was $\sim 15 \mathrm{~dB}$ lower than for similarly sedated guinea pigs. Thus, it is clear the species' difference seen in the $f_{1}$-constancy tests is mainly a result of differences in acoustic reflex thresholds. While sectioning the rabbit's middle-ear muscles should eliminate the acoustic reflex, such a lesion also eliminated DPOAEs in the 1-day recovered animal (Martin, unpublished observations) presumably because of the reduced tension on the middle-ear structures.

Future research will establish if this average efferent measure and associated $f_{1}$-constancy test can be modified for use in humans to detect differences in innate efferent activity that might predict susceptibility to noise overexposure, or perhaps even detect brainstem disorders that involve the central auditory nervous system (Liberman \& Guinan 1998; Berlin et al. 1994).

\section{ACKNOWLEDGMENTS}

This work was supported by the Public Health Service (DC03086, DC03114, DC00613) and the University of Miami's Chandler Chair Fund. We would like to thank Dr. Brenda Lonsbury-Martin and Dr. Glen Martin and the reviewers for their suggestions to this article.

\section{REFERENCES}

Astl J, Popelar J, Kvasnak E, Syka J. Comparison of response properties of neurons in the inferior colliculus of guinea pigs under different anesthetics. Audiology 35:335-345, 1996.

Berlin CI, Hood LJ, Hurley A, Wen H. The First Jerger Lecture. Contralateral suppression of otoacoustic emissions: an index of the function of the medial olivocochlear system. Otolaryngol. Head Neck Surg. 110:3-21, 1994.

BROWN AM. Acoustic distortion from rodent ears: A comparison of responses from rats, guinea pigs and gerbils. Hear. Res. 31:2538, 1987.

Chery-Croze S, Moulin A, Collet L. Effect of contralateral sound stimulation on the distortion product $2 f_{1}-f_{2}$ in humans: evidence of a frequency specificity. Hear. Res. 68:53-58, 1993.

Collet L, Kemp DT, Veuillet E, Duclaux R, Moulin A, Morgon A. Effect of contralateral auditory stimuli on active cochlear micromechanical properties in human subjects. Hear. Res. 43:251261, 1990

GUINAN JJ JR, WARR WB, NORRIS BE. Differential olivocochlear projections from lateral versus medial zones of the superior olivary complex. J. Comp. Neurol. 221:358-370, 1983.

GUINAN JJ JR, WARR WB, NORRIS BE. Topographic organization of the olivocochlear projections from the lateral and medial zones of the superior olivary complex. J. Comp. Neurol. 226:21-27, 1984.

Harel N, Kakigi A, Hirakawa H, Mount RJ, Harrison RV. The effects of anesthesia on otoacoustic emissions. Hear. Res. 110:2533, 1997.

Liberman MC, GUINAN JJ JR. Feedback control of the auditory periphery: anti-masking effects of middle ear muscles vs. olivocochlear efferents. J Commun Disord 31:471-553, 1998.

Liberman MC, PURIA S, GUINAN JJ JR. The ipsilaterally evoked olivocochlear reflex causes rapid adaptation of the $2 f_{1}-f_{2}$ distortion product otoacoustic emission. J. Acoust. Soc. Am. 99:35723584, 1996.

MAISON SF, LibERMAN MC. Predicting vulnerability to acoustic injury with a noninvasive assay of olivocochlear reflex strength. J. Neurosci. 20:4701-4707, 2000.

Martin GK, Jassir D, Stagner BB, Whitehead ML, LonsburyMARTIN BL. Locus of generation for the $2 f_{1}-f_{2}$ vs $2 f_{2}-f_{1}$ distortion-product otoacoustic emissions in normal-hearing humans revealed by suppression tuning, onset latencies, and amplitude correlations. J. Acoust. Soc. Am. 103:1957-1971, 1998.

Moulin A, Collet L, Duclaux R. Contralateral auditory stimulation alters acoustic distortion products in humans. Hear. Res. 65:193-210, 1993

Mountain DC, Geisler CD, Hubbard AE. Stimulation of efferents alters the cochlear microphonic and the sound-induced resistance changes measured in scale media of the guinea pig. Hear. Res. 3:231-240, 1980.

Puel JL, Rebillard G. Effect of contralateral sound stimulation on the distortion product $2 F_{1}-F_{2}$ : evidence that the medial efferent system is involved. J. Acoust. Soc. Am. 87:1630-1635, 1990.

SIEGEL JH, KIM DO. Efferent neural control of cochlear mechanics? 
Olivocochlear bundle stimulation affects cochlear biomechanical nonlinearity. Hear. Res. 6:171-182, 1982.

SMith DI, Mills JH. Anesthesia effects: auditory brain-stem response. Electroencephalogr. Clin. Neurophysiol. 72:422-428, 1989.

Sun XM, KIM DO. Adaptation of $2 f_{1}-2 f_{2}$ distortion product otoacoustic emission in young-adult and old CBA and C57 mice. J. Acoust. Soc. Am. 105:3399-3409, 1999.
WHITEHEAD ML, LONSBURY-MARTIN BL, MARTIN GK. Slow variation of the amplitude of acoustic distortion at $f_{2}-f_{1}$ in awake rabbits. Hear. Res. 51:293-300, 1991.

Whitehead ML, LONSBURY-MarTin BL, MarTin GK. Evidence for two discrete sources of $2 f_{1}-f_{2}$ distortion-product otoacoustic emission in rabbit: I. Differential dependence on stimulus parameters. J. Acoust. Soc. Am. 91:1587-1607, 1992. 